\title{
Réagencements territoriaux et conduites des politiques sociales à l'échelle locale
}

Territorial re-organisation at a local lever and social policies governance:

decentralization from downwards

\section{Agnès Gramain et Samuel Neuberg}

\section{OpenEdition}

\section{Journals}

Édition électronique

URL : http://journals.openedition.org/travailemploi/1717

DOI : 10.4000/travailemploi. 1717

ISSN : 1775-416X

Éditeur

DARES - Ministère du Travail

Édition imprimée

Date de publication : 30 septembre 2009

Pagination : $77-87$

ISSN : 0224-4365

Référence électronique

Agnès Gramain et Samuel Neuberg, «Réagencements territoriaux et conduites des politiques sociales à l'échelle locale », Travail et Emploi [En ligne], 119 | juillet-septembre 2009, mis en ligne le 30

septembre 2011, consulté le 20 avril 2019. URL : http://journals.openedition.org/travailemploi/1717 ; DOl : 10.4000/travailemploi. 1717 


\title{
Réagencements territoriaux récents et conduite des politiques sociales à l'échelle locale
}

\author{
Agnès Gramain (*), Samuel Neuberg( **)
}

\begin{abstract}
L'enquête européenne "Rescaling Social Welfare Policies. A comparative study on the path towards multi-level governance in Europe" avait pour objectif de comparer, entre différents pays européens, les processus de réagencement territorial dans la conduite des politiques sociales. Les enquêtes de terrain réalisées pour cette recherche sur quatre sites français, ont apporté, sur la "deuxième vague de décentralisation" (2004-2005), des informations riches de détails difficiles à faire entrer dans les cadres simples prévus pour la comparaison internationale. Ainsi, ce que l'on nomme "décentralisation" apparaît plutôt comme un mélange de mouvements contraires, selon le point de vue adopté (vue du haut ou du bas) et les fonctions considérées (planification, financement, définition des populations cibles...). Les réagencements à l'œuvre ont, sur la régulation de l'action publique, des conséquences ambiguës. Ils font de la régulation par la concurrence un modèle particulièrement séduisant en théorie. Cependant, les conditions de sa mise en auvre concrète par les collectivités territoriales (l'étroitesse du "marché» ou la nouveauté des missions pour le donneur d'ordre par exemple) remettent en cause sa pertinence et son efficacité. Dans ce contexte contradictoire s'instaure un jeu de négociations locales permanentes qui influence les pratiques des opérateurs en matière de sélection des publics et conduit ce faisant à une disparité dans les réponses apportées aux populations prises en charge aussi bien entre territoires que selon le point d'entrée institutionnel à l'intérieur d'un territoire donné.
\end{abstract}

L'enquête européenne "Rescaling Social Welfare Policies. A comparative study on the path towards multi-level governance in Europe"(1) (2) (voir encadré 1) avait pour objectif de comparer, entre différents pays européens, les processus de réagencement territorial dans l'élaboration et la mise en œuvre des politiques sociales. Les analyses de comparaison internationale se sont révélées, sans grande surprise, très difficiles à mener à un niveau de précision satisfaisant. Au-delà des difficultés évidentes posées par l'hétérogénéité des découpages territoriaux et des champs de politique sociale d'un pays à l'autre, une difficulté plus radicale est apparue. Pour rendre opérationnelle une comparaison internationale avec les ressources matérielles de l'équipe «rescaling» il était inévitable de la caler

(*) Bureau d'économie théorique et appliquée, université de Nancy 2, université de Strasbourg, CNRS; agnes.gramain@ univ-nancy2.fr.

(**) Centre Maurice Halbwachs, ENS, EHESS, CNRS; neuberg@clipper.ens.fr.

(1) La participation de la France à cette enquête a été initiée par la DARES et conduite par une équipe de chercheurs comprenant, outre les auteurs de l'article, J. Billois, A. Exertier, J.-M. Herbillon et M. Lexton.

(2) Dans la suite du texte, il sera fait référence à cette recherche par le terme «rescaling» seul. sur les définitions institutionnelles des dispositifs et des acteurs de leur mise en ouvre. Mais dès le recueil du matériau (voir encadré 2), les résultats se sont révélés très difficiles à interpréter dans ces cadres institutionnels et ont laissé entrevoir d'autres découpages et d'autres modes de fonctionnements pertinents. Dans le même temps, la dimension très ethnographique de l'enquête réalisée en France (Gramain, Herbillon, Neuberg, 2008) a mis en lumière l'intérêt d'une analyse moins centrée sur l'intervention publique telle qu'elle est définie par la loi, mais d'avantage sur les formes concrètes de l'intervention publique et sur leur relation avec les définitions légales qui les régulent.

Une lecture d'ensemble des réponses fournies par les professionnels de terrain à nos interrogations sur la réponse publique apportée localement à différents cas-types (3) est très instructive: pour la plupart des cas-types utilisés, il est impossible de trouver deux professionnels ayant donné des réponses similaires. Des différences sont perceptibles jusque dans les aspects des dispositifs les plus encadrés par la

(3) Cette méthode de recueil était désignée, en vocabulaire européen, sous le terme de «vignettes». 


\section{Encadré 1 \\ Méthodologie de la recherche "Rescaling social policies in Europe"}

Le projet de recherche «rescaling" impliquait, outre la France, des équipes de recherche issues de huit pays(1), l'équipe autrichienne assurant la coordination d'ensemble. L'objectif était d'analyser les disparités en matière de gouvernance des politiques sociales et d'évolution de cette gouvernance, à l'échelle de l'Europe entre pays différents, mais aussi à l'échelle de chaque pays entre territoires différents. Quatre secteurs d'intervention ont été explorés: accompagnement des chômeurs, lutte contre la pauvreté, prise en charge de la dépendance et immigration(2). Du point de vue du matériau analysé, le projet s'appuyait tout d'abord sur une description standardisée des cadres et des dispositifs nationaux. Cette première phase était complétée par deux enquêtes spécifiques conduites chacune sur deux sites dans chaque pays. L'une permettait de recueillir une description de l'organisation concrète de la réponse des pouvoirs publics à l'échelle d'un bénéficiaire potentiel, à partir de castypes présentés à différents opérateurs en charge de la mise en œuvre des dispositifs. La deuxième enquête devait, à partir d'entretien-experts avec des responsables publics, schématiser le fonctionnement d'ensemble de l'action publique, à l'échelle d'un territoire, grâce à des tableaux synoptiques standardisés précisant l'implication de chaque échelon territorial dans différentes fonctions prédéfinies (planification, définition des critères d'éligibilité, financement...). Cette méthodologie "européenne" a été complétée, pour la France, par la réalisation de quatre monographies locales (Gramain, HerBiLlon, Neuberg, 2008).

(1) Suisse, Espagne, Italie, Pologne, Finlande, Suède, Norvège. (2) Ce quatrième secteur n'avait pas été retenu pour la participation française.

loi, d'un territoire à l'autre mais tout autant d'un professionnel à l'autre, en fonction de la place qu'il occupe dans l'architecture d'ensemble des dispositifs. Dans une perspective comparatiste, la tentation est grande d'homogénéiser les réponses obtenues à la lumière des textes et définitions officielles. Nous choisissons ici la démarche inverse: il s'agit pour nous de réinterpréter les réponses au questionnaire à la lumière des observations de terrain pour tenter de donner sens à cette profusion.

Notre matériau empirique est donc un sousproduit de l'enquête originale et le cœur de l'analyse développée est un effet secondaire des difficultés rencontrées dans la construction de la comparaison internationale. Les incohérences apparentes qui tenaient en échec les tentatives de standardisation des données françaises selon les modalités prévues par l'enquête européenne sont ici utilisées comme point de départ pour une réinterprétation des transformations récentes de la mise en œuvre des poli- tiques publiques, et notamment la «seconde vague de décentralisation $»$ de 2004-2005.

Cette démarche implique donc d'abord de revenir sur les notions qui ont présidé au réagencement territorial des politiques publiques. Qu'elles désignent des processus, comme la «décentralisation», ou des formes de structuration, comme le «maillage» du territoire, elles imposent une lecture «par le haut» des situations de terrain et laissent échapper une part cruciale des transformations à l'œuvre. Le décalage entre ces catégories institutionnalisées et la situation réelle telle que peut l'objectiver l'enquête conduit à s'interroger sur les formes concrètes de régulation du système, tendu entre des définitions légales très contraignantes et des logiques pratiques de fonctionnement qui n'y sont pas réductibles. Cette reconstruction des logiques pratiques des différents acteurs permet alors finalement de restituer les formes de cohérence interne à l'intervention publique et de comprendre la manière dont ces fonctionnements de terrain coproduisent jusqu'aux caractéristiques les plus profondes et les plus «politiques » des dispositifs d'intervention publique.

\section{Les changements d'échelle territoriale}

Avant même de s'intéresser aux spécificités proprement territoriales de la mise en œuvre des politiques sociales en France, il apparaît clairement que la dichotomie «centralisation / décentralisation» ne saurait saisir de façon précise les réorganisations récentes des différents champs d'intervention. Ainsi, le processus communément appelé «seconde vague de décentralisation» a mis en jeu des transferts de compétences et de responsabilités généralement à double sens.

\section{Des réagencements à double sens}

Parmi les champs de politique sociale explorés, celui de la prise en charge des personnes âgées dépendantes est le meilleur exemple de cette simultanéité de tendances contraires. Concernant la planification de l'action gérontologique, la tendance récente est à la décentralisation, non seulement dans les textes, mais dans l'exercice réel de la fonction: les conseils généraux se sont clairement saisis de leur rôle de chef de file dans les quatre sites d'enquête. En revanche une tendance inverse se dessine tout aussi généralement pour ce qui est des financements et de la définition des critères d'accès aux aides publiques.

Les mécanismes de «recentralisation » sont différents dans ces deux cas. Pour ce qui est du financement, c'est la création de la Caisse nationale de solidarité pour l'autonomie (CNSA) qui est en jeu. Près de $90 \%$ des financements publics relèvent en fait 


\section{Encadré 2 \\ Cadres analytiques}

Le matériau recueilli a été analysé en croisant regards de sociologie et d'économie sur les transformations en cours.

\section{Les outils d'analyse de la sociologie pour les monographies}

Nous avons mobilisé deux grands types d'approches sociologiques.

Un premier grand ensemble de travaux analyse ce que l'ethnographie contemporaine appelle l'«économie domestique " (WEBER, 2006), terme repris de l'anthropologie économique. Ce courant s'attache à une observation fine des pratiques économiques des acteurs, sans présupposé sur l'échelle pertinente de la prise de décision ni sur la finalité des comportements. Il apparaît d'une part que les agents sont capables d'une grande finesse stratégique, et d'un intense travail de rationalisation de leurs comportements économiques (ZeLIZER, 1995). En revanche, l'ethnographe est immédiatement confronté à la prolifération des formes du raisonnement beaucoup plus vaste que ne le suppose la théorie économique standard: les acteurs mettent en œuvre des modes différenciés de prise de décision économique, selon qu'ils décident seuls ou collectivement, qu'il s'agit d'investir un capital ou soutenir un proche, que la situation est banale ou exceptionnelle (GoJARD et al. 2003). Ce corps de recherches permet l'étude de l'impact local des réorganisations des politiques publiques, à partir d'un constat fondamental: les différents dispositifs n'ont d'existence qu'imbriqués, pour former un paysage dense de contraintes, de ressources et d'incitations dans lequel professionnels et bénéficiaires se meuvent.

Le second type d'approche s'attache à comprendre comment les politiques sociales produisent des institutions économiques. Elles établissent d'abord des catégorisations qui servent de point de repères dans la prise de décision économique. Ainsi le fonctionnement du marché du travail est-il dépendant des dispositifs publics qui opèrent des appariements sélectifs entre les emplois vaquant et les chômeurs (Demażère, 2000). Plus largement, ces dispositifs solidifient des représentations collectives. Dans le domaine des politiques de l'emploi, l'adaptation des dispositifs à des situations de chômage durable a contribué à cimenter la notion d' "exclusion sociale" (PAUGam, 2004), modifiant les rôles du travail dans les définitions des individus (CASTEL, 1999). Enfin, les politiques portant sur la «qualité de vie» des personnes dépendantes ont contribué à fixer ou à diffuser les normes sociales et économiques du comportement. Dans tous les cas, ces normes sont l'objet d'une négociation perpétuelle au cours de laquelle se reconstruisent et se déplacent les frontières entre groupes sociaux (DESROZIÈRES, 2000).

\section{Les outils d'analyse de la science économique}

L'analyse économique jette sur le réagencement territorial des politiques publiques un regard essentiellement normatif: il s'agit de savoir à quel échelon de gouvernement (local ou fédéral) confier la décision concernant la provision des biens publics pour obtenir une allocation efficace des ressources. Ce champ de l'analyse économique, dont on attribue traditionnellement l'ouverture à Tiebout (TIEBOUT, 1956), recourt à des concepts et des raisonnements classiques de la microéconomie de la deuxième moitié du XXe siècle (MıGNOLET, 2005). La décentralisation est vue, par exemple, comme un mécanisme révélateur qui contre les comportements de passagers clandestins dans le financement des biens publics: dès lors que les administrés choisissent leur lieu de résidence en fonction de leur appréciation de la politique locale, l'existence d'une palette de politiques locales conduit les individus à une mobilité résidentielle qui révèlent leurs préférences et permet aux pouvoirs publics de les mettre à contribution efficacement pour financer les programmes locaux d'action publique. La centralisation, quant à elle, est présentée comme un moyen de mutualiser plus efficacement les politiques ayant une composante assurantielle ou de réduire l'existence d'externalités géographiques. Enfin, le partage des financements entre gouvernements fédéral et local s'apparente à une règle mixte issue d'un arbitrage entre assurance et incitation.

Bien que consacré aux réagencements territoriaux, le projet de recherche «rescaling» se prêtait mal à la mobilisation de ce type d'approche, non seulement parce qu'il relevait essentiellement d'une démarche positive (il s'agissait avant tout de décrypter les réagencements effectivement à l'œuvre) mais aussi parce que le matériau se composait de sites ponctuels à comparer et non d'une vue d'ensemble d'une structure territoriale, verticale ou horizontale. De ce fait, la démarche retenue repose essentiellement sur l'application des concepts de l'analyse standard de la régulation économique à l'action publique locale.

De manière générale, en l'absence de documentation proprement historique sur les différents contextes locaux, les témoignages des acteurs sur le passé local ont ici toujours été analysés comme des conséquences des transformations en cours et non comme des explications causales.

aujourd'hui de la solidarité nationale, dès lors que l'on explicite les transferts de la CNSA aux conseils généraux. Plus important, la répartition territoriale des financements est aujourd'hui en grande partie décidée par la CNSA qui affecte directement des enveloppes à l'échelon départemental, aussi bien pour le financement de l'Allocation personnalisée d'autonomie (APA) (l'enveloppe est alors transférée aux conseils généraux) que pour le financement des établissements d'hébergement (l'enveloppe est alors transférée aux directions départementales de l'action sanitaire et sociale). Dans la pratique, 
la politique d'ouverture de lits en établissement apparaît ainsi totalement soumise aux décisions de financement de l'échelon central.

Pour ce qui est des critères d'accès aux aides publiques, le mécanisme de centralisation est plus indirect. En effet, si l'APA est un dispositif national, les conseils généraux ne sont contraints que par des montants «plancher» et restent maîtres du vaste secteur des aides facultatives. Cependant, nos interlocuteurs du conseil général soulignent que les obligations légales qui leur sont imposées et leur poids budgétaire induisent indirectement d'importantes contraintes sur le reste de leur action, dont la dimension facultative. La part la moins centralisée tend donc à se réduire, aux marges des cadres nationaux.

Ce résultat suggère que les transferts de compétence de l'État aux collectivités ne s'accompagnent pas nécessairement d'une décentralisation de l'ensemble des fonctions, telles que l'affectation des financements ou la définition des catégories de bénéficiaires, par exemple.

Même en limitant l'analyse à une fonction donnée des pouvoirs publics, le transfert de compétence de l'État central aux collectivités territoriales, processus qu' on pourrait qualifier de «décentralisation », peut conduire, dans les faits, à une organisation de l'action publique plus centralisée, si on la considère depuis l'échelon infradépartemental.

La «décentralisation du RMI» illustre, dans certains territoires, ce problème crucial du point de vue adopté, notamment à travers la modification du rôle des Centres communaux d'action sociale (CCAS) en tant qu'instructeurs des demandes et accompagnateurs des bénéficiaires. Sur le site B, la réorganisation de l'accompagnement des bénéficiaires du RMI par le conseil général a posé de grandes difficultés. Le conseil général et l'Union Départementale des CCAS ont travaillé en 2005 sur un projet de convention cadre qui n'a jamais abouti. L'enjeu du conflit illustre l'importance d'une analyse des transferts de compétence, non seulement «vu du haut», c'est-à-dire de l'échelon le plus large, mais aussi «vu du bas». Les CCAS demandent en effet que la charge de travail inhérente à l'accompagnement des bénéficiaires du RMI soit prise en compte par le conseil général et que celui-ci finance des postes supplémentaires. Le conseil général préfère de son côté créer des postes dans ses propres structures. Dans ce cas l'État central transfère aux conseils généraux une compétence dont la mise en œuvre était en fait jusque-là déléguée à un échelon territorial inférieur à celui du département; ce transfert se traduit alors par une «re-centralisation» de l'action publique du point de vue du citoyen, dès lors que le conseil général la territorialise à un échelon plus large que celui des anciens délégataires : leur interlocuteur n'est plus le CCAS dans le cas du RMI mais les unités territoriales du département.

\section{Des structures territoriales multiples à l'échelle infradépartementale}

Le transfert des compétences par bloc à des collectivités territoriales sans relations hiérarchiques induit qui plus est des besoins de coordination particuliers. En effet, plusieurs compétences transférées à des exécutifs différents doivent souvent être mobilisées simultanément pour répondre à une même situation. Or, chaque exécutif et chaque institution concernée par l'action publique dispose de ses propres relais aux différents échelons territoriaux. Les acteurs publics à l'échelle d'un territoire départemental regroupent donc non seulement les directions du conseil général, mais aussi les représentations départementales du conseil régional, ainsi que les directions départementales de l'État central, sans compter l'ensemble des autres institutions concernées, ANPE (4), Assedic, AFPA, agences régionales d'hospitalisation, sécurité sociale, etc. (BENARRosh, 2000).

La structure d'ensemble reste relativement simple aux échelons régional et départemental, puisque l'ensemble des institutions s'organise grosso modo selon ce découpage administratif. En revanche, l'inscription territoriale de l'action publique devient particulièrement variable et complexe dès lors que l'on aborde l'échelon infradépartemental. En effet, les découpages politiques des cantons et des arrondissements ne semblent pas servir de référence. Chaque institution invente des territoires «locaux» qui lui sont propres, le maillage choisi pouvant varier d'un site à l'autre, d'un champ de politique sociale à l'autre, voire même d'une institution à l'autre pour un même site et un même champ d'intervention.

Ainsi, alors que sur le site $\mathrm{B}$, les maillages territoriaux sont à peu près cohérents, sur le site $\mathrm{A}$, la communauté d'agglomérations regroupe douze communes situées dans deux départements différents, appartenant à deux régions différentes. La ville centre a son propre CCAS. Tous les autres découpages s'inscrivent dans les frontières départementales, sans cohérence avec la communauté d'agglomération, qu'il s'agisse de la zone emploiformation (la ville centre et une commune de banlieue), des unités territoriales du conseil général, de la mission locale (deux morceaux de communautés de communes), du plan local d'insertion (une commune extérieure), ou encore de la zone d'emploi qui s'étend sur trois communautés, mais n'en englobe aucune entièrement.

Si la décentralisation conduit à une certaine diversité géographique en matière d'organisation territoriale de l'action publique, c'est donc essentiellement à l'échelon le plus fin: en fonction des choix faits par les collectivités territoriales et les

(4) À la date de l'enquête, la fusion entre l'ANPE et les Assedic n'était encore qu'en projet. 
acteurs du champ, les degrés de cohérence et d'emboîtement des différents maillages peuvent varier.

L'enquête réalisée révèle une deuxième particularité de l'organisation locale de l'action publique en France: le pouvoir de proposition et d'élaboration des schémas d'action publique, à défaut du pouvoir proprement décisionnel, est bien souvent confié à des comités. La liste des comités cités dans les entretiens est impressionnante et ceux-ci sont de natures extrêmement variables. Certains sont constitués par la loi, d'autres sont d'initiative locale, ce qui d'ailleurs ne présage en rien de leur réelle activité.

La conduite de la politique gérontologique départementale sur le site D fournit l'exemple le plus achevé que nous ayons rencontré dans le cadre de notre enquête, d'un fonctionnement par comités locaux : le suivi de l'APA, l'élaboration et la signature des conventions tripartites avec les établissements d'hébergement pour personnes âgées, la rédaction et le suivi du schéma gérontologique, sont confiés à des comités dont le périmètre varie mais qui reposent toujours sur un même tandem de tête composé par la direction des affaires sociales du conseil général et la DDASS. De l'avis de l'ensemble des participants, ces comités constituent des outils réels de conduite de l'action publique, et permettent les concertations tant techniques que stratégiques. L'efficacité de ces comités est d'ailleurs soulignée par des observateurs extérieurs: la Cour des comptes via sa chambre régionale a ainsi conclu, dans son dernier rapport, que «le département [...] semble s'être doté d'instruments pertinents pour suivre le schéma gérontologique sur la période et, à ce jour, ces instruments fonctionnent».

Il convient cependant de noter que cette particularité française de la coordination locale de l'action publique complique singulièrement l'analyse de l'exercice réel du pouvoir: d'un point de vue méthodologique, ces comités opèrent comme un écran. La liste officielle des membres d'un comité ne dit rien ni de l'activité ni de l'influence plus ou moins importante des uns et des autres. L'analyse des disparités territoriales dans la gouvernance des politiques locales ne peut donc se contenter d'une comparaison des structures mises en place «sur le papier».

\section{Réagencements territoriaux et modes de régulation de l'action publique}

Les conséquences des réagencements territoriaux à l'œuvre dans la conduite des politiques sociales dépassent la simple question de la coordination entre pouvoirs publics. En effet, les modes de régulation de l'action publique, et en particulier des opérateurs chargés de la mise en œuvre, apparaissent eux aussi modelés par les nouvelles organisations territoriales.

\section{La mise en concurrence comme modèle de référence}

Les entretiens réalisés montrent l'omniprésence du modèle d'une régulation par la mise en concurrence. Cette tendance a bien sûr une composante juridique avec l'entrée, à partir de 2003, des contrats de délégation de service public signés par les exécutifs locaux dans le code des marchés publics de 2001. À cela s'ajoute une nécessité pratique de déléguer: l'État central a en effet dévolu brutalement aux collectivités locales des missions de gestion et de diagnostic qu'elles ne pouvaient matériellement pas assumer sans délai, aussi bien pour la gestion du RMI que pour celle de l'APA, par exemple. Elles ont donc opté massivement pour un recours à la prestation de service. Enfin, au-delà de cette nécessité pratique, dans un climat de grande pression sur les exécutifs locaux pour garantir une «bonne gestion» de l'immense afflux d'argent public décentralisé, le modèle d'une régulation autonome par la concurrence a pu apparaître comme une solution ingénieuse. Ce modèle permet en effet de déléguer la mise en ouvre à des opérateurs tout en déléguant leur contrôle à la mécanique concurrentielle elle-même.

Du point de vue de l'organisation concrète, la volonté de recourir à une logique de régulation par un quasi-marché(5), apparaît à travers trois thématiques principales : le renforcement de la distinction entre opérateurs et prescripteurs, le développement des procédures de sélection par appel d'offre et enfin les modes de rémunération (voir encadré 3 ).

\section{Du discours à la pratique}

Cependant, et c'est là toute l'ambiguïté, en même temps qu'il s'affirme comme perspective dominante en matière de régulation des politiques publiques, le modèle concurrentiel apparaît largement remis en cause, dans sa pertinence et dans son efficacité (Mougeot, Naegelen, 1993), dès lors que l'on envisage les conditions de sa mise en œuvre dans la conduite des politiques sociales par les collectivités territoriales. Ainsi, quand l'enquête se déplace de la description des procédures officielles d'attribution des marchés publics et d'élaborations de partenariats entre institutions vers l'observation des relations concrètes et quotidiennes entre acteurs, on obtient un tableau d'ensemble bien différent.

(5) On entend par quasi-marché une situation de concurrence organisée permettant d'obtenir un résultat similaire à celui d'une concurrence marchande (appel d'offre, concurrence fictive dans le secteur hospitalier...). 


\section{Encadré 3}

\section{Éléments d'une régulation par la mise en concurrence}

\section{Le découpage des fonctions}

Dans le cadre de la réorganisation de l'accompagnement des bénéficiaires du RMI, le conseil général sur le site $\mathrm{A}$ a confié à un tiers associatif la mission de réaliser les bilans-diagnostics et de proposer un référencement, mission réalisée jusque-là par les organismes en charge du suivi lui-même, comme le CCAS par exemple.

Sur le site $\mathrm{C}$, où le service social départemental pour les personnes âgées était délégué, depuis fort longtemps, à une association offrant par ailleurs un service d'aide à domicile, le conseil général a choisi, afin de respecter la loi(1), "de mettre fin à la délégation confiée aux organismes et associations en matière de service social gérontologique» (rapport du président du conseil général relatif au budget primitif 2007) et d'assurer lui-même cette fonction, non sans quelques difficultés pratiques et quelques remous locaux (Gramain, Herbillon, Neuberg, 2008).

\section{La sélection des prestataires}

La référence à la sélection par appel d'offre est très répandue, sauf dans le champ de la prise en charge de la dépendance (2): sur l'ensemble des sites d'enquête, les Assedic mettent en place des appels d'offre pour leurs opérations de formation et les conseils généraux sélectionnent ainsi les organismes référents pour les bénéficiaires du RMI pour certaines catégories de bénéficiaires; sur le site A, même les actions mises en place dans le cadre du plan local pour l'insertion et l'emploi (PLIE) sont pour une part sélectionnées par appel d'offre.

\section{Les modes de financement}

Sur le site A., le conseil régional paie les organismes de formation au service fait sur facture mensuelle et retient une réserve de $5 \%$ versée après observation du devenir des stagiaires à l'issue de la formation. Dans le cadre du PLIE, sur le même site, $60 \%$ du budget initial est versé sur vérification des objectifs de moyens; l'association gestionnaire du PLIE fait ensuite une proposition de solde, à l'issue du contrôle final, tenant compte des objectifs de résultats (taux de sorties positives) et de critères qualitatifs.

(1) La loi prévoit explicitement que l'équipe de professionnels chargée de l'évaluation de la dépendance dans l'instruction du dossier de demande d'APA ne peut être la même que celle qui fournit les soins financés par l'allocation.

(2) De manière surprenante, la régulation de l'offre en direction des personnes âgées échappe totalement à la référence concurrentielle. Les procédures de labellisation, de conventionnement, de négociation, se font certes sur la base de projets proposés par les offreurs, projets qui sont évalués à l'aune des priorités inscrites dans les schémas et les programmations. Mais les différents projets ne sont pas présentés comme étant en compétition directe, malgré l'existence d'un calendrier qui restreint les quelques moments où les projets d'ouverture de lits peuvent être acceptés (les "fenêtres" dans le vocabulaire technique).

Les procédures de sélection par appel d'offre sont loin d'être systématiques. À cela plusieurs raisons. Tout d'abord, la mise en place d'appels d'offre suppose de pouvoir établir un cahier des charges pertinent. Or les collectivités territoriales ne se sentent pas toujours la maîtrise de telles compétences. Ensuite, la variété des statuts des commanditaires de l'action publique complique singulièrement l'organisation d'appels d'offre. En ce qui concerne la formation des demandeurs d'emploi par exemple, les deux financeurs principaux, les Assedic et les conseils régionaux, soulignent souvent l'intérêt qu'il y aurait à articuler les deux types de financements pour une même formation afin d'atteindre une certaine masse critique. Mais tandis que les conseils régionaux relèvent du code des marchés publics, les Assedic sont des organismes de droit privé(6). Enfin, la situation côté «prestataires» n'est pas toujours plus propice à l'organisation d'une sélection concurrentielle. D'une part certains

(6) Le même type de difficulté se retrouve dans le cas de l'insertion par l'activité économique: la sélection des prestataires ne peut relever des marchés publics puisqu'elle implique toujours d'autres financeurs. opérateurs s'avèrent incontournables : l'AFPA dans le champ de la formation, mais aussi l'ANPE ou les CCAS dans la désignation des référents pour les bénéficiaires du RMI, occupent une position institutionnelle unique, souvent héritée de l'organisation antérieure. Par surcroît, même abstraction faite de ces cas particuliers, les pouvoirs publics sont souvent contraints par le petit nombre de structures susceptibles de répondre à leur demande. Ce petit nombre peut tenir à l'histoire locale: ainsi, sur le site $\mathrm{C}$, seules quatre associations interviennent localement dans l'aide à la prise en charge des personnes âgées dépendantes, et l'une d'entre elle concentre plus de $90 \%$ de l'activité (en 2003, elle assurait 911057 heures sur les 998000 réalisées au total). Il peut être plus structurel, lorsqu'il tient à l'étroitesse du marché sur lequel opèrent certaines structures ayant des compétences spécifiques. Sur le site A par exemple, comme le précise explicitement le directeur adjoint de l'insertion du conseil général, le suivi des populations spécifiques (SDF, populations gitanes, personnes handicapées, personnes sortant de prison...) dans le cadre de la gestion du RMI a été confié par convention à des structures associatives, habilitées par le président du conseil général, 
et non par appel d'offre, car le nombre de structures pouvant réaliser de telles missions était trop faible. On peut même aller jusqu'à penser que l'accompagnement de bénéficiaires, quels qu'ils soient, fait de leurs référents des experts sans alternative. Pour une part, les interventions déléguées dans ce champ de politique publique font, par leur nature même, obstacle à l'existence de la concurrence.

Les modalités de financement des prestataires retenus par les pouvoirs publics apparaissent aussi assez éloignées du schéma théorique décrit dans un premier temps par nos interlocuteurs. Selon eux, l'instauration de la loi organique relative aux lois de finances (LOLF) conduit à introduire des obligations de résultats, mais se contenter de ce critère est présenté comme trop risqué lorsque le résultat échappe en partie aux prestataires. C'est évidemment dans le secteur de l'insertion professionnelle que cet argument est le plus net, en particulier pour les prestataires qui travaillent avec des personnes en grande difficulté.

Non seulement les pouvoirs publics manquent souvent d'alternatives locales, mais en auraient-ils une que l'application de financements au résultat risquerait de la détruire. Comme l'ont souligné plusieurs de nos interlocuteurs, les opérateurs sont eux-mêmes très dépendants de la commande publique et fragiles budgétairement: ne payer qu'au service fait induit des difficultés de trésorerie qui peuvent être insurmontables pour les structures, ne pas sélectionner un prestataire revient mécaniquement à le mettre en difficulté. Comme l'exprime sans détour le directeur d'une unité territoriale au sein du conseil général (site $\mathrm{A})$ : «On est pas dans une relation purement marchande [...]. Il y a une dépendance mutuelle quand même (souligné par nous). C'est pour ça aussi que ça équilibre les choses. Il n'y a pas d'un côté un commanditaire tout puissant et de l'autre côté des artisans oppressés. Il y a un commanditaire, il y a un prestataire. Ils sont dans un système qui est quand même "co", donc un système de dépendance.»

La décentralisation semble donc jouer un rôle ambigu sur le mode de régulation des opérateurs en charge de l'action publique à l'échelle locale. $\mathrm{Si}$ elle fait de la régulation concurrentielle un modèle particulièrement séduisant, par sa capacité à assurer l'usage efficace des deniers publics de manière autonome, elle en dévoie la mise en œuvre, non seulement parce que le type de missions déléguées est chargé d'incertitude et d'informations spécifiques, mais aussi parce que l'échelle territoriale conduit à des situations de face à face entre un unique acheteur et un unique prestataire. Les rapports réels entre acteurs apparaissent de fait plus proches d'un modèle de négociation généralisée très loin des fonctionnements théoriques de marché (CROZIER, 1971).

\section{L'impact des réagencements territoriaux sur les disparités de mise en œuvre des politiques sociales}

L'objectif de l'enquête «rescaling» était d'analyser les disparités en matière de gouvernance des politiques sociales et non de résultat de l'action publique. Cependant, même si la structure du matériau recueilli ne permet pas de porter un diagnostic tranché sur ce point (encadré 4), l'enquête a mis au jour plusieurs mécanismes générateurs de disparités entre bénéficiaires à l'intérieur d'un territoire donné, mécanismes qui ne sont pas sans lien avec les réagencements territoriaux et la gouvernance locale ainsi que les modes de régulation qui les accompagnent.

\section{La multiplicité des points d'entrée dans les dispositifs d'aide}

La mise en place de régulations formellement marchandes n'a pas seulement conduit à rendre difficiles d'approche les négociations incessantes entre le petit nombre d'acteurs concernés. Elle a aussi abouti à une multiplication des points d'entrée dans les différents dispositifs pour un même territoire. Ainsi par exemple sur le site $\mathrm{C}$, la mise en œuvre de la politique départementale en direction des personnes âgées était traditionnellement déléguée à une association unique. Mais la décentralisation d'une partie des compétences de l'État au conseil général et l'instauration d'un mécanisme formel de concurrence ont conduit le conseil général à se tourner vers d'autres prestataires «concurrents». Sans se prononcer sur la modification éventuelle de l'efficacité globale du système sur ce territoire, il est clair que le nombre d'interlocuteurs potentiels d'un futur bénéficiaire s'est sensiblement accru.

Cependant, la lutte contre les positions locales de monopole ne peut suffire à expliquer une caractéristique frappante de la situation sur le terrain: la coexistence de «guichets» dépendant d'acteurs de niveaux différents. Ceci est particulièrement net dans la gestion du RMI. Dès la création du dispositif en 1988, l'instruction des demandes avait été très largement déléguée, non seulement aux CCAS mais aussi à un nombre important d'associations travaillant au contact de publics spécifiques. Cette dissémination était perçue par les pouvoirs publics (LeLIÈvRE, 2008) comme le moyen d'assurer la couverture la plus large possible, y compris aux «marges» de la société. Sur tous les sites de l'enquête, le nouvel acteur fort, le conseil général, a tenté de construire ou de renforcer ses propres possibilités d'accueil direct du public, sans avoir pour autant les moyens de revenir, au moins dans un premier temps, sur les délégations «traditionnelles» aux CCAS et associations. Mais ces derniers ont très générale- 


\section{Encadré 4}

\section{Un matériau à utiliser avec précaution pour analyser les disparités et leur génèse}

L'enquête «rescaling" portait sur les disparités en matière de gouvernance et non en matière de résultat de l'action publique pour les bénéficiaires. Pour autant le matériau recueilli apporte quelques éclairages sur ce point: on peut en effet tenter de repérer dans la diversité des modalités de mise en œuvre des politiques sociales à l'échelon local, décrite via les cas-types, les éléments porteurs d'une disparité dans l'offre et la prise en charge des publics concernés. L'analyse du matériau doit cependant se faire avec prudence, pour quatre raisons.

- Tout d'abord, le recueil sur les conséquences pour les bénéficiaires n'est qu'indirect.

- Ensuite, l'analyse est contrainte par le choix des sites d'enquête. Ils sont tout d'abord peu nombreux, ce qui interdit toute généralisation à la France dans son ensemble. Les comparaisons portent en outre sur des chaînes de pouvoirs publics entièrement différentes: les communes sont situées dans des départements différents, euxmêmes situés dans des régions différentes. Ceci empêche d'une part d'isoler l'effet propre à chaque niveau de pouvoir public; ceci rend d'autre part les disparités internes à un territoire difficiles à percevoir.

- Le recueil de données a été effectué à partir de vignettes (cas-types). Ce protocole a un inconvénient évident: il ne permet d'obtenir qu'un discours «en moyenne» ou plus exactement un discours normatif. Un deuxième inconvénient du protocole est apparu grâce aux observations et entretiens conduits en marge de la passation des vignettes. La construction des cas-types à partir des critères d'éligibilité des différents dispositifs d'aide ne permet d'observer que les disparités qui tiennent à ces critères. Or, il semble que la disparité des trajectoires des bénéficiaires tienne tout autant à des catégorisations infralégales, reposant sur des caractéristiques individuelles bien distinctes de celles qui apparaissent dans les textes de lois (et donc dans les cas-types).

- Enfin, le matériau recueilli ne permet pas de comparer systématiquement les disparités observables au sein des territoires avant et après la deuxième vague de décentralisation. Ceci étant, cette limite est probablement incontournable, puisqu'il ne peut exister de site «témoin » n'ayant pas connu cette modification institutionnelle, si tant est que la notion de situation contre-factuelle s'applique ici.

ment cherché à préserver leur propre structure d'accueil du public, sans se contenter des missions d'accompagnement des bénéficiaires(7). Les réformes 2004-2005 ont donc finalement conduit à la création d'un niveau de guichet supplémentaire.

\section{Effet du point d'entrée sur la réponse des pouvoirs publics}

Cette multiplication des «guichets» induite par la décentralisation n'est pas sans conséquence sur la réponse publique proposée aux difficultés d'un bénéficiaire.

L'enquête révèle en effet que cette réponse n'est pas indépendante du type et de la position locale des institutions qui ont «accueilli » le bénéficiaire dans chacun des dispositifs le concernant. La «non-neutralité » du point d'entrée dans les différents dispositifs publics s'explique par deux types de limites. D'abord s'imposent des limites cognitives à la maîtrise des rouages des dispositifs par les professionnels de terrain. La méthodologie des «vignettes» est ici particulièrement révélatrice. Elles mettent brutalement en évidence combien les connaissances techniques des professionnels concernant les différentes sous-parties de leur champ d'intervention peuvent varier, en fonction notamment de la place qu'eux-mêmes y occupent. Ceux-ci ne font d'ailleurs pas mystère de leur senti-

(7) Le même phénomène est également observable, quoique de façon plus localisée, dans le champ des politiques gérontologiques, en particulier dans la relation entre les CLIC et les pôles gérontologiques des conseils généraux. ment d'être fréquemment dépassés par la multiplication des dispositifs ad hoc en renouvellement constant, et cela d'autant plus que les règles actuelles en matière de délégation de service publique impliquent une redéfinition régulière des cahiers des charges. La seconde limite tient à la perception qu'ont les professionnels d'un fort rationnement des moyens (Delfini, Demazière, 2000). Celle-ci induit en effet une sursélection des bénéficiaires potentiels des mesures les plus coûteuses, sursélection d'autant plus drastique que le bénéficiaire s'est adressé à un guichet moins bien placé pour défendre «ses» dossiers. Les critères réels de cet écrémage sont ainsi variables d'une institution à l'autre et nécessairement moins transparents que la définition légale des populations éligibles (BENARROSH, 2006).

La prise en charge des bénéficiaires du RMI offre un exemple très clair de l'effet combiné de ces deux logiques. En plus du RMI proprement dit, les bénéficiaires peuvent prétendre par exemple à la fois aux aides facultatives municipales en tant qu'ils sont démunis, et aux emplois aidés en tant qu'ils sont chômeurs. En comparant les réponses apportées aux questions des vignettes, on observe que lorsque le premier contact avec le dispositif RMI s'opère par l'intermédiaire d'un CCAS, le bénéficiaire se voit très souvent proposer des aides municipales, comme l'aide alimentaire, mais a peu de chance d'être même clairement informé des possibilités de candidature à un emploi aidé. S'il est entré dans le dispositif RMI suite à une prise en charge par le Service public de l'emploi, le résultat est en général inverse. 
Ainsi, même si le matériau utilisé suggère de rester prudent, on constate que le transfert de compétences aux conseils généraux s'accompagne de fortes disparités internes aux territoires considérés, disparités qui tiennent à la nouvelle variété des points d'entrée ainsi qu'aux réaménagements locaux des critères de sélection qu'encouragent les nouveaux modes de régulation des opérateurs.

\section{Le rôle des catégorisations implicites dans la réponse des pouvoirs publics}

Indépendamment des enjeux normatifs, l'existence d'une multitude d'opérations de catégorisation implicite des bénéficiaires par les acteurs locaux de la mise en œuvre des politiques publiques mérite attention. Ces catégories fonctionnent comme points de repères cognitifs pour les professionnels et structurent la dimension stratégique de leur pratique.

Ces deux points sont particulièrement visibles, dans l'ensemble des champs d'intervention, quand se pose la question des transferts budgétaires liés aux différentes délégations de service public et à l'évaluation de la qualité du délégataire. La population cible de l'intervention est toujours définie comme une catégorie ou un ensemble de catégories de bénéficiaires supposés homogènes, auquel est liée une évaluation du coût de la prise en charge et de ses objectifs qualitatifs. Mais dans la pratique, ces catégories regroupent systématiquement des situations variées qui échappent au découpage légal. Dans ces conditions, la double pression à la maîtrise des coûts et à l'amélioration des résultats n'est tenable qu'à condition que ces caractéristiques «cachées » de la population cible ne compliquent pas trop la mise en œuvre des dispositifs. Cette situation explique d'ailleurs largement la multiplication des «guichets» décrite précédemment: garder, via un guichet, une prise sur le flux de bénéficiaires entrant permet à un acteur d'influencer en partie les caractéristiques «cachées» des bénéficiaires orientés vers «ses» dispositifs et de garder ainsi un contrôle minimal sur les conditions économiques réelles de son activité(8).

Il convient de souligner à quel point ces classifications pratiques des types de bénéficiaires et de dispositifs jouent un rôle fondamental de coordination entre acteurs. La simple question de la prise en charge des publics «spécifiques», qui cumulent les «problématiques» ou requièrent l'intervention d'acteurs spécialisés, suppose des formes de division du travail inexprimables dans les catégories légales (JeLLAB, 1997). La tendance très présente

(8) On retrouve ici un fonctionnement largement documenté dans le cas de l'attribution des budgets hospitaliers en fonction d'une nomenclature de groupes de malades dits homogènes, qui a conduit les États-Unis à légiférer sur les sélections des patients (NEwHOUSE, 1996). sur le terrain à la séparation des bénéficiaires du RMI entre un groupe relevant de l'insertion professionnelle et un autre destiné à une prise en charge «sociale» illustre parfaitement les enjeux de ces processus. Sur le site A, une gestionnaire d'étape du PLIE évoque par exemple la manière dont les professionnels opèrent une sélection des «problématiques» en fonction de la logique pratique de leur intervention, toujours plus cadrée que la définition légale de leur rôle: «est-ce qu'on a pas tendance à mettre des ceillères sur un problème d'alcool? [...] on va l'ignorer parce que ben ça nous arrange pas des masses. Si on commence à perdre deux mois à parler d'alcool... ». Cette distinction pratique n'est évidemment pas sans rapport avec l'accroissement des exigences quantitatives portant sur le secteur de l'insertion professionnelle. En fait, l'effet des politiques publiques sur le terrain dépend de ces arrangements locaux, en l'occurrence la mise en retrait des «problématiques» non professionnelles et la sélection des bénéficiaires qu'elle suppose. La maîtrise opérationnelle que le «chef de file» détient sur ces opérations de sélection implicite est un élément décisif de son pouvoir réel sur le champ d'intervention.

Le caractère stratégique pour les acteurs de ces actes de catégorisation pratique explique partiellement leur caractère implicite. Leur invisibilité tient aussi, plus structurellement, à la forme générale prise en France par le réagencement territorial de la mise en œuvre des politiques publiques, i.e. une décentralisation de la mise en œuvre concrète des politiques, articulée au maintien d'une définition centralisée des catégories de bénéficiaires et des types de dispositifs, dans une logique d'égalité entre territoires. Cette architecture d'ensemble tend à rendre indicible les formes de catégorisations locales qui constituent, par définition, autant de ruptures de «l'égalité républicaine».

Cette analyse dégage un constat empirique fort, conséquence directe de l'introduction d'une régulation formellement concurrentielle dans le secteur de l'aide sociale, simultanée en France du processus de «décentralisation» proprement dite, bien qu'elle en soit théoriquement distincte. D'une part, sans pouvoir juger d'un éventuel accroissement des disparités globales dans le traitement des bénéficiaires, ces changements des formes de régulation du secteur aboutissent au creusement de disparités nouvelles entre les bénéficiaires. Celles-ci jouent principalement à l'intérieur des différents territoires plutôt qu'entre eux, et elles ne correspondent pas au modèle traditionnel de classement moral des «pauvres». Si ces critères moraux du jugement n'ont pas disparu, ils sont largement recouverts par des considérations liées à l'efficacité immédiate des dispositifs. Le destin anticipé du bénéficiaire semble dominer aujourd' hui tout autre critère d'orientation. D'autre part, cette réorganisation rend difficile l'interprétation des écarts entre les normes légales et le 
traitement réel des bénéficiaires. Ces «déviances» sont en effet essentiellement le produit des tentatives des acteurs de terrain de rationaliser leur propre pratique. En utilisant des formes de distinction extra-légales entre bénéficiaires, les professionnels de la prise en charge accroissent paradoxalement la cohérence du secteur. Sans être dénué d'efficacité sur le terrain, ce fonctionnement ne peut qu'amener à s'interroger sur le décalage qu'il crée structurellement entre le lieu du débat politique de fond sur les missions des pouvoirs publics et celui de l'élaboration d'une grande partie des logiques pratiques de leur intervention.

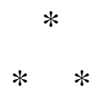

L'analyse des informations de terrain recueillies aux marges du protocole de l'enquête "rescaling» et des difficultés méthodologiques rencontrées dans la comparaison internationale des réagencements territoriaux récents dans le champ des politiques sociales montre donc que les rapports entretenus effectivement entre les pouvoirs publics et les différents opérateurs de l'action publique, à l'échelle locale, relèvent plutôt d'un modèle de négociation généralisée, bien loin des fonctionnements théoriques d'une régulation marchande ou quasi- marchande. Dans ces négociations, la possibilité de définir et d'appliquer des critères pratiques de sélection des bénéficiaires pour leur orientation vers les différents dispositifs d'aide constitue sans nul doute un des enjeux majeurs. La localisation réelle des marges de manœuvre qui résulte de ces négociations latentes détermine des configurations locales très diverses d'un point à l'autre du territoire. Le rôle de la diversité de ces configurations dans les disparités de mise en œuvre des politiques sociales entre les territoires, mais aussi à l'intérieur de chacun d'entre eux, mériterait d'être étudié de manière plus systématique. En effet, le matériau exploité ici ne permet au mieux que de fournir quelques pistes mais pas d'explorer systématiquement la variété des réagencements locaux durant la seconde vague de décentralisation ni de mesurer leurs effets sur l'ampleur des disparités dans la mise en œuvre des politiques sociales. Ce matériau suggère cependant que les configurations locales ne sont pas des montages hétéroclites et a priori inefficaces, bien qu'elles s'instaurent dans un implicite sans coordination apparente: dans les réagencements fins des circuits locaux de prise en charge et des critères pratiques qui en gouvernent l'accès semblent en effet se dessiner des orientations de fond perçues comme telles par les acteurs, qui permettent la coordination entre ce grand nombre d'acteurs hétérogènes. C'est alors la question du lieu réel de la décision politique qui se trouve posée, puisque, ces catégories pratiques dérogeant au caractère impératif des définitions légales de l'action publique, elles restent inexprimables dans la sphère publique. 


\section{Bibliographie}

Benarrosh Y. (2000), «Tri des chômeurs: le nécessaire consensus des acteurs de l'emploi », Travail et Emploi, $\mathrm{n}^{\circ} 81$.

Benarrosh Y. (2006), Recevoir les chômeurs à l'ANPE. L'institution entre don et contrat, L'Harmattan.

Boltanski L., Chiapello E. (1999), Le nouvel esprit du capitalisme, NRF.

Castel R. (1999), Les métamorphoses de la question sociale, Gallimard.

Crozier M. (1971), Le phénomène bureaucratique, Seuil.

Delfini C., Demazière D. (2000), «Le traitement de l'offre d'emploi à l'ANPE: diversité des logiques d'intermédiation», Travail et Emploi, $\mathrm{n}^{\circ} 81$.

DemazièRe D. (2000), «Jugement d'employabilité et construction sociale du chômage», in Gradey J. (dir.), Emploi et travail, regards croisés, L'Harmattan.

DesRosières A. (2000), La politique des grands nombres. Histoire de la raison statistique, La Découverte.

Gramain A., Herbillon J.-M., Neuberg S. (2008), Rescaling social welfare policies in Europe: rapport final, rapport de recherche remis à la Dares, juillet 2008.

Jellab A. (1997), Le travail d'insertion en mission locale, L'Harmattan.

Legay A., Monchatre S. (2000), «L'ANPE à l'épreuve du local», Travail et Emploi, $\mathrm{n}^{\circ} 81$.
Lelièvre M., Nauze-Fichet E. (dir.) (2008), RMI, l'état des lieux, 1988-2008, La Découverte.

L'Horty Y., AnNe D. (2002), «Transferts sociaux locaux et retour à l'emploi », Économie et Statistique, n 357 358.

Mignolet M. (ed.) (2005), Le fédéralisme fiscal, coll. «Économie société région», De Boeck.

Mougeot M., Naegelen F. (1993), Les marchés publics, Economica.

Newhouse J.-P. (1996), "Reimbursement Health Plans and Health Providers: Efficiency in production versus selection", Journal of Economic Literature, vol. 34, n 3.

Paugam S. (2004), Disqualification sociale, essai sur la nouvelle pauvreté, PUF.

Tiebout C.-M. (1956), “A Pure Theory of Local Expenditures", The Journal of Political Economy, vol. 64, $\mathrm{n}^{\circ} 5$.

Weber F., Gojard S., Gramain A. (dir.) (2003), Charges de famille, dépendance et parenté dans la France contemporaine, La Découverte.

Weber F. (2006), L'économie domestique, Aux lieux d'être.

Zelizer V. A. (1995), The social meaning of money, Basic books.

Zelizer V. A. (2007), The purchase of intimacy, Princeton University Press. 